\title{
MHD Forced Convection Laminar Boundary Layer Flow of Alumina-Water Nanofluid over a Moving Permeable Flat Plate with Convective Surface Boundary Condition
}

\author{
S. M. AbdEl-Gaied and M. A. A. Hamad \\ Mathematics Department, Faculty of Science, Assiut University, Assiut 71516, Egypt \\ Correspondence should be addressed to S. M. AbdEl-Gaied; sagaied123@gmail.com
}

Received 17 July 2013; Revised 4 August 2013; Accepted 8 August 2013

Academic Editor: Mohamed Fathy El-Amin

Copyright (C) 2013 S. M. AbdEl-Gaied and M. A. A. Hamad. This is an open access article distributed under the Creative Commons Attribution License, which permits unrestricted use, distribution, and reproduction in any medium, provided the original work is properly cited.

\begin{abstract}
This paper analyses a two-dimensional steady forced convection boundary layer viscous incompressible flow of alumina-water nanofluid over a moving permeable vertical flat plate under the effect of a magnetic field normal to the plate. Thermal convective surface boundary condition is applied. The nanofluid formulated in the present study is water dispersed with various volumetric fractions of the alumina $\left(\mathrm{Al}_{2} \mathrm{O}_{3}\right)$ nanoparticles. The plate velocity and the free stream velocities are considered to be proportional to $x^{n}$, while the magnetic field and suction velocities are taken to be proportional to $(x)^{(n-1) / 2}$. The similarity solution of the governing problem is obtained. Numerical studies are presented to show the effect of the nanoparticle volume fraction $\phi$, the convective heat transfer parameter $b$, the power law exponent $n$, the wall velocity parameter $A$, and the suction parameter $f_{w}$ on the velocity, temperature, skin-friction coefficient, and Nusselt number.
\end{abstract}

\section{Introduction}

It is known that nanofluids can tremendously enhance the heat transfer characteristics of the original (base) fluid. Thus, nanofluids have many applications in industry such as coolants, lubricants, heat exchangers, and microchannel heat sinks. Therefore, numerous methods have been taken to improve the thermal conductivity of these fluids by suspending nano-/microsized particle materials in liquids. Several recent studies on the modeling of free/mixed convection heat transfer in nanofluids have been published: Congedo et al. [1], Ghasemi and Aminossadati [2], and Ho et al. [3, 4]. These studies have used traditional finite difference and finite volume techniques with the tremendous call on computational resources that these techniques necessitate. Hamad et al. [5] used the application of a one-parameter group to present similarity reductions for problems of magnetic field effects on free-convection flow of a nanofluid past a semi-infinite vertical flat plate. Hamad and Pop [6] studied theoretically the similarity solution of the steady boundary layer flow near the stagnation-point flow on a permeable stretching sheet in a porous medium saturated with a nanofluid and in the presence of internal heat generation/absorption. Hamad [7] found the analytical solutions of convective flow and heat transfer of an incompressible viscous nanofluid past a semi-infinite vertical stretching sheet in the presence of magnetic field. Abu-Nada and Chamkha [8] investigated the natural convection heat transfer characteristics in a differentially heated enclosure filled with a CuO-EG-water nanofluid for different published variable thermal conductivity and variable viscosity models. Teng et al. [9] studied the effect of particle size, temperature, and weight fraction on the thermal conductivity ratio of $\mathrm{Al}_{2} \mathrm{O}_{3}$-water nanofluid. Ho et al. [10] presented an experimental study for natural convection heat transfer of a nanofluid in vertical square enclosure of different sizes. Bachok et al. [11] studied the steady boundary-layer flow of a nanofluid past a moving semi-infinite flat plate in a uniform free stream. Hady et al. [12] studied the natural convection boundary-layer flow over a downward-pointing vertical cone in a porous medium saturated with a non-Newtonian nanofluid in the presence of heat generation or absorption. The numerical modeling 
of the conjugate heat transfer and fluid flow of $\mathrm{Al}_{2} \mathrm{O}_{3} /$ water nanofluid through the microchannel heat sink was presented by Lelea [13]. In this paper, the laminar flow regime was considered along with viscous dissipation effect. Also, the microchannel heat sink with square microchannels and $\mathrm{Dh}=$ $50 \mu \mathrm{m}$ was considered. Nandy and Mahapatra [14] analyzed the effects of velocity slip and heat generation/absorption on magnetohydrodynamic (MHD) stagnation-point flow and heat transfer over a stretching/shrinking surface, with convective boundary conditions, in the presence of nanoparticle fractions. They considered also the effects of Brownian motion and thermophoresis. Numerical study for the effects of thermal radiation and viscous dissipation on hydromagnetic mixed convection flow over a nonlinear stretching and shrinking sheets in nanofluids was presented by Pal et al. [15]. Rohni et al. [16] theoretically investigated the steady two-dimensional boundary-layer flow past a moving semiinfinite flat plate in a water-based nanofluid containing three different types of nanoparticles: copper (cuprum), alumina (aluminium oxide), and titania (titanium dioxide). For more information, see also Das et al. [17], Wang and Mujumdar [1820], and Kakaç and Pramuanjaroenkij [21].

Heat transfer due to forced convection occurs in many areas. Examples include chemical process, biochemical process, and engineering. Its study is important due to the need to improve the efficiency of the heat transfer. Ali [22] studied the effect of temperature dependent viscosity on laminar mixed convection boundary layer flow and heat transfer on a continuously moving vertical isothermal surface and obtained local similarity solutions. According to previous studies, the forced convection flow over moving plate has many practical engineering applications such as liquid films in condensation process and in aerodynamics. Similarity solutions about moving plate were investigated by many authors. Among them, Ishak et al. [23] studied the boundary layer flow on a moving permeable plate parallel to a moving stream and concluded that dual solutions exist if the plate and the free stream move in the opposite directions. Magyari [24] studied the moving plate thermometer with uniform velocity. His approach was based on a series solution of the problem using the Merkin transformation method. Hoernel [25] investigated similarity solution for steady laminar boundary layer equations governing MHD flow near forward stagnation point of two dimensional moving axisymmetric bodies. Recently, Pal and Mondal [26] studied MHD forced convection and heat transfer of an incompressible Newtonian electrically conducting and heat generating/absorbing fluid having temperature-dependent viscosity over a nonisothermal permeable wedge in the presence of thermal radiation. In all of the above studies, convectional thermal boundary conditions (either prescribed heat flux or prescribed surface temperature) were used.

According to previous scholars, the MHD flow has received the attention of many researchers due to its engineering applications. In metallurgy, for example, some processes involve the cooling of many continuous strips by drawing them through an electrically conducting fluid subject to a magnetic field (Kandasamy and Muhaimin [27]). This allows the rate of cooling to be controlled and final product with the desired characteristics to be obtained. Another important application of hydromagnetic flow in metallurgy is in the purification of molten metals from nonmetallic inclusions through the application of a magnetic field. Research has also been carried out by previous researchers on the flow and heat transfer effects of electrically conducting fluids such as liquid metals, water mixed with a little acid and other equivalent substances in the presence of a magnetic field. The studies have involved different geometries and different boundary conditions. Herdricha et al. [28] studied MHD flow control for plasma technology applications. They identified potential applications for magnetically controlled plasmas in the fields of space technology as well as in plasma technology. Seddeek et al. [29] investigated the similarity solution in MHD flow and heat transfer over a wedge taking into account variable viscosity and thermal conductivities. Alam et al. [30] investigated the effects of variable suction and thermophoresis on steady MHD combined free-forced convective heat and mass transfer flow over a semi-infinite permeable inclined plate in the presence of thermal radiation. Aydin and Kaya [31] studied MHD mixed convection of a viscous dissipating fluid about a permeable vertical flat plate and found that the value of Richardson number determines the effect of the magnetic parameter on the momentum and heat transfer. Recently, Rahman and Salahuddin [32] investigated the effects of variable electric conductivity and viscosity on hydromagnetic heat and mass transfer flow along a radiated isothermal inclined permeable surface in a stationary fluid which includes internal heat generation.

The aim of the present study is to analyze steady, two-dimension, laminar MHD forced convection flow of alumina-water nanofluid on moving permeable flat plate with convective surface boundary condition. The similarity representation of the partial differential equations governing this problem is obtained. The motivation of this study is to explore the influence of nanofluid parameters with the effect of suction, convective heat transfer, and velocity parameter. The variation distribution of the shear stress and heat transfer rates (the physical quantities of interest in this study) with the parameters governing the problem are presented.

\section{Formulation of the Problem}

Consider a two-dimensional forced convective flow of waterbased alumina nanoparticles over a moving permeable flat plate. A rectangular coordinate frame is selected in which the $x$-axis is vertical and $y$-axis is horizontal. A magnetic field of variable strength $B(x)$ is applied perpendicular to the plate. The temperature of the plate on the top is held at a uniform temperature $T_{w}$ which is assumed greater than the ambient temperature $T_{\infty}$. It will be further assumed that the bottom surface of the plate is heated by convection from a hot fluid of temperature $T_{f}$, and this generates a heat transfer coefficient $h_{f}$ (see Aziz [33]). For the thermophysical properties of the alumina, see Oztop and AbuNada [34]. The flow configuration and the coordinate system are shown in Figure 1. Kinematic viscosity and thermal diffusivity are assumed constants. Particle coagulation, the magnetic Reynolds number, the electric field owing to the 


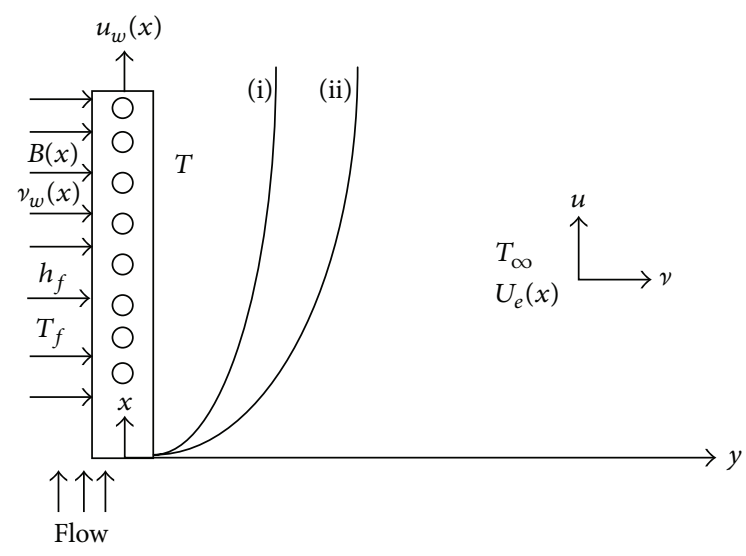

(i) Thermal B.L.

(ii) Momentum B.L

FIGURE 1: Flow configuration and coordinate system.

polarization of charges, viscous dissipation term, and Hall effects are assumed to be negligible. The field variables of this study are the velocity $\vec{V}=(u, v)$ and the temperature $T$. Under the above assumptions, the boundary layer equations can be written in dimensional form as (see Schlichting and Gersten [35], Kays and Crawford [36])

$$
\begin{gathered}
\frac{\partial u}{\partial x}+\frac{\partial v}{\partial y}=0 \\
\rho_{\mathrm{nf}}\left(u \frac{\partial u}{\partial x}+v \frac{\partial u}{\partial y}\right)=-\frac{\partial p}{\partial x}+\mu_{\mathrm{nf}} \frac{\partial^{2} u}{\partial y^{2}}-\sigma B^{2}(x) u \\
u \frac{\partial T}{\partial x}+v \frac{\partial T}{\partial y}=\alpha_{\mathrm{nf}} \frac{\partial^{2} T}{\partial y^{2}} .
\end{gathered}
$$

The corresponding boundary conditions are

$$
\begin{gathered}
u=u_{w}(x), \quad v=-v_{w}(x), \\
-\kappa_{\mathrm{nf}} \frac{\partial T}{\partial y}=h_{f}\left[T_{f}-T_{w}\right] \quad \text { at } y=0, \\
u \longrightarrow U_{e}(x), \quad T \longrightarrow T_{\infty}, \quad C \longrightarrow C_{\infty} \quad \text { as } y \longrightarrow \infty .
\end{gathered}
$$

Here, $p$ is the pressure, $\sigma$ is the electric conductivity, $U_{e}(x)$ is potential velocity, $u_{w}(x)$ is the velocity of the moving plate, $v_{w}(x)$ is the velocity normal to the plate with $v_{w}(x)>0$ for injection (blowing) and $v_{w}(x)<0$ for suction, and $v_{w}(x)=0$ corresponds to an impermeable sheet. As usual $\mu_{\mathrm{nf}}$ is the nanofluid viscosity, $\rho_{\mathrm{nf}}$ is the nanofluid density, $\alpha_{\mathrm{nf}}$ is the nanofluid thermal diffusivity and, $\kappa_{\mathrm{nf}}$ is the nanofluid thermal conductivity, which are given by

$$
\begin{aligned}
& \mu_{\mathrm{nf}}=\frac{\mu_{f}}{(1-\phi)^{2.5}}, \quad \alpha_{\mathrm{nf}}=\frac{\kappa_{\mathrm{nf}}}{\left(\rho C_{p}\right)_{\mathrm{nf}}}, \\
& \left(\rho C_{p}\right)_{\mathrm{nf}}=(1-\phi)\left(\rho C_{p}\right)_{f}+\phi\left(\rho C_{p}\right)_{s},
\end{aligned}
$$

$$
\begin{gathered}
\rho_{\mathrm{nf}}=(1-\phi) \rho_{f}+\phi \rho_{s}, \\
\kappa_{\mathrm{nf}}=\frac{\kappa_{f}\left(\kappa_{s}+2 \kappa_{f}-2 \phi\left(\kappa_{f}-\kappa_{s}\right)\right)}{\left(\kappa_{s}+2 \kappa_{f}+\phi\left(\kappa_{f}-\kappa_{s}\right)\right)},
\end{gathered}
$$

where $\phi$ is the nanoparticle volume fraction, $\left(\rho C_{p}\right)_{\mathrm{nf}}$ is the nanofluid heat capacity, $\kappa_{f}$ and $\kappa_{s}$ are thermal conductivities of the fluid and of the solid fractions, respectively, and $\rho_{f}$ and $\rho_{s}$ are the densities of the fluid and of the solid fractions, respectively.

In the free stream, $u=U_{e}(x)$, and so (2) reduces to

$$
U_{e} \frac{d U_{e}}{d x}=-\frac{1}{\rho_{\mathrm{nf}}} \frac{\partial p}{\partial x}-\frac{\sigma B^{2}(x)}{\rho_{\mathrm{nf}}} U_{e}(x) .
$$

Eliminating the pressure gradient term $\partial p / \partial x$ between (2) and (6), we get

$$
\begin{aligned}
u \frac{\partial u}{\partial x}+v \frac{\partial u}{\partial y}= & U_{e} \frac{d U_{e}}{d x} \\
& +\frac{1}{\rho_{\mathrm{nf}}}\left[\mu_{\mathrm{nf}} \frac{\partial^{2} u}{\partial y^{2}}-\sigma B^{2}(x)\left(u-U_{e}\right)\right]
\end{aligned}
$$

By introducing stream function $\psi$, such that

$$
u=\frac{\partial \psi}{\partial y}, \quad v=-\frac{\partial \psi}{\partial x} .
$$

We also introduce the following dimensionless transformations:

$$
\begin{gathered}
\eta=\frac{y}{x} \sqrt{n+1} \operatorname{Re}_{x}^{1 / 2}, \quad \psi=\frac{v_{f}}{\sqrt{n+1}} \operatorname{Re}_{x}^{1 / 2} f(\eta), \\
\theta(\eta)=\frac{T-T_{\infty}}{T_{f}-T_{\infty}}, \quad \operatorname{Re}_{x}=\frac{U_{\infty} x^{n+1}}{v_{f}}, \\
B(x)=B_{0} x^{(n-1) / 2}, \quad u_{w}(x)=u_{0} x^{n} \\
v_{w}(x)=v_{0} x^{(n-1) / 2}, \quad U_{e}(x)=U_{\infty} x^{n},
\end{gathered}
$$

where $B_{0}, u_{0}, v_{0}$, and $u_{\infty}$ are constants and where $\mathrm{Re}_{x}$ is the Reynolds number based on position $x$ along flow direction (see Hoernel [25]). Here, $n$ (not necessarily an integer) is the power law exponent, $u_{0}>0$ means that the plate is moving along the positive direction of $x$-axis, and $u_{0}<0$ means that the plate is moving in the negative direction of $x$-axis. In our analysis, we choose $u_{0}>0$. The quantity $v_{0}$ represents suction or injection, $v_{0}>0$ represents suction, and $v_{0}<0$ represents injection. 
It is noticed that the continuity equation (1) is satisfied automatically and (3) and (7) yield

$$
\begin{aligned}
& \frac{n+1}{(1-\phi)^{2.5}} f^{\prime \prime \prime} \\
& \quad+\left(1-\phi+\left(\frac{\rho_{s}}{\rho_{f}}\right) \phi\right) \\
& \quad \times\left[\frac{n+1}{2} f f^{\prime \prime}-n f^{\prime 2}+n\right]+M\left(f^{\prime}-1\right)=0, \\
& \frac{1}{\operatorname{Pr}}\left(\frac{\kappa_{\mathrm{nf}}}{\kappa_{f}}\right) \theta^{\prime \prime}+\frac{1}{2}\left(1-\phi+\frac{\left(\rho C_{p}\right)_{s}}{\left(\rho C_{p}\right)_{f}} \phi\right) f \theta^{\prime}=0,
\end{aligned}
$$

where $\operatorname{Pr}=\left(\mu c_{p}\right)_{f} / \kappa_{f}$ is the Prandtl number and $M=$ $\sigma B_{0}^{2} /\left(\rho_{f} u_{\infty}\right)$ is the magnetic parameter. Here, we assume that $T_{f}>T_{w}>T_{\infty}$.

The corresponding boundary conditions in (4) become

$$
\begin{gathered}
f^{\prime}(0)=A, \quad f(0)=f_{w}, \\
\theta^{\prime}(0)=-b\left(\frac{\kappa_{f}}{\kappa_{\mathrm{nf}}}\right)[1-\theta(0)], \quad f^{\prime}(\infty)=1, \quad \theta(\infty)=0,
\end{gathered}
$$

where $A=u_{0} / U_{\infty}$ is a constant and is considered as a velocity parameter and $f_{w}=-2 v_{0} / \sqrt{v U_{\infty}(n+1)}$ is the suction parameter. The parameter $A>0$ means that the plate is moving in the same direction as the free stream velocity, $A<0$ means that the plate is moving in the opposite direction of the free steam, and $A=0$ stands for static plate.

The parameter $b=\left(h_{f} / \kappa\right) \sqrt{v x / U_{e}(n+1)}$ is the convective heat transfer parameter, which should be constant. To fulfill this criterion, the coefficient of heat transfer $h_{f}$ must be directly proportional to $1 / \sqrt{x}$; that is, $h_{f}=c_{1} / \sqrt{x}$.

\subsection{Particular Cases}

(i) Here, we should mention that if $n=0$ (the full stream velocity is constant), $f_{w}=0$ (the plate is impermeable), $u_{w}=0$ (the plate is stationary), and $M=0$ (absence of magnetic field), our system (10)-(12) reduces to that in Aziz [33].

(ii) If $f_{w} \neq 0$ (the plate is permeable), $u_{w}=0$, and $M=0$, our system (10)-(12) reduces to that in Ishak [37].

The parameters of engineering interest for the problem are the skin friction $C_{f}$ and the local Nusselt number $\mathrm{Nu}$, respectively. These physical quantities can be derived from the following definitions:

$$
\begin{gathered}
C_{f}=\frac{\mu_{\mathrm{nf}}}{u_{w}^{2} \rho_{\mathrm{nf}}}\left(\frac{\partial u}{\partial y}\right)_{y=0}, \\
\mathrm{Nu}=\frac{-x}{\left(T_{f}-T_{\infty}\right)}\left(\frac{\kappa_{\mathrm{nf}}}{\kappa_{f}}\right)\left(\frac{\partial T}{\partial y}\right)_{y=0} .
\end{gathered}
$$

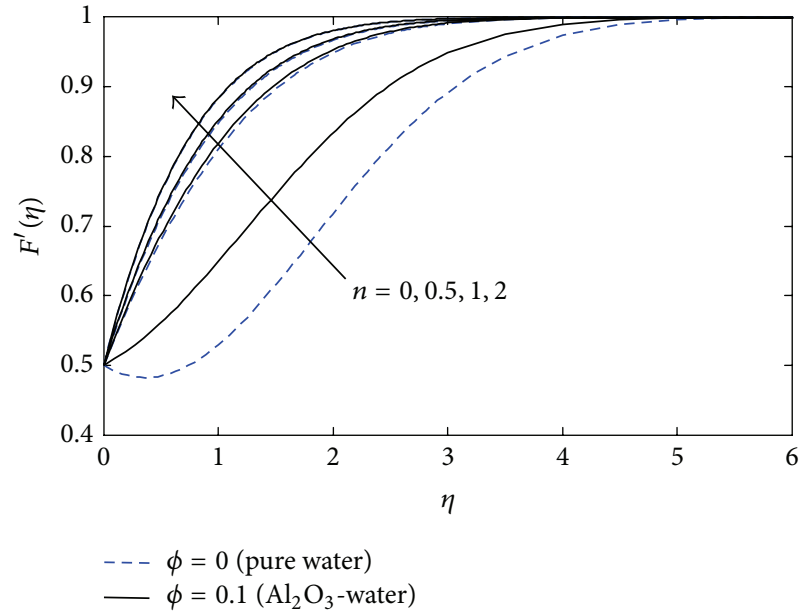

Figure $2: b=0.5, f_{w}=0.5, \operatorname{Pr}=6.2, M=0.5$, and $A=0.5$.

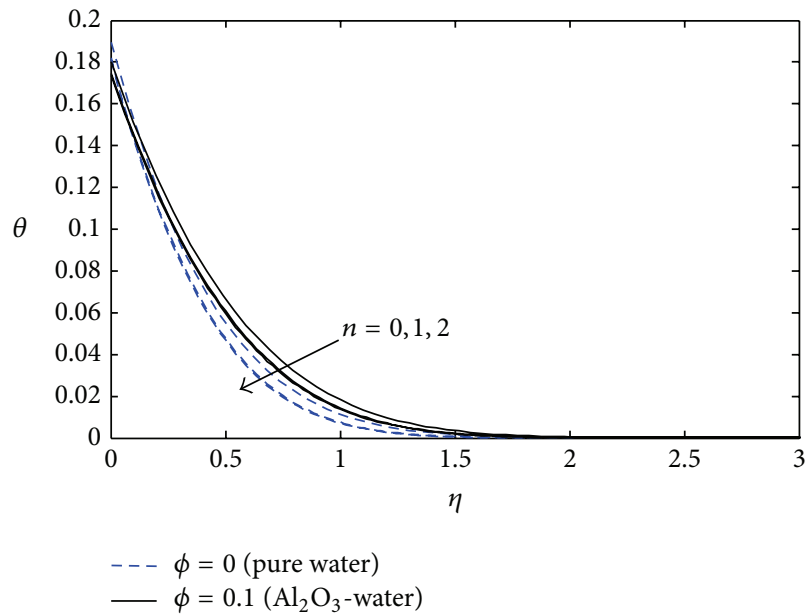

Figure $3: b=0.5, f_{w}=0.5, \operatorname{Pr}=6.2, M=0.5$, and $A=0.5$.

By substituting from (8) and (12) into (13), we get

$$
\begin{aligned}
& \operatorname{Re}_{x}^{1 / 2} C_{f}=\frac{\sqrt{n+1}}{A^{2}(1-\phi)^{2.5}} f^{\prime \prime}(0), \\
& \operatorname{Re}^{-1 / 2} \mathrm{Nu}=-\sqrt{n+1} \frac{\kappa_{\mathrm{nf}}}{\kappa_{f}} \theta^{\prime}(0) .
\end{aligned}
$$

\section{Results and Discussion}

The transformed system of nonlinear ordinary differential equations (10) and (11) together with boundary conditions (12) containing various parameters, namely, the power law parameter $n$, magnetic parameter $M$, the Prandtl number $\operatorname{Pr}$, the nanoparticles volume fraction $\phi$ and convective heat transfer parameter $b$, suction parameter $f_{w}$ and the velocity parameter $A$. Equations (10) and (11) together with boundary conditions given in (12) are solved numerically by an implicit finite difference method. To exhibit our results obtained by 


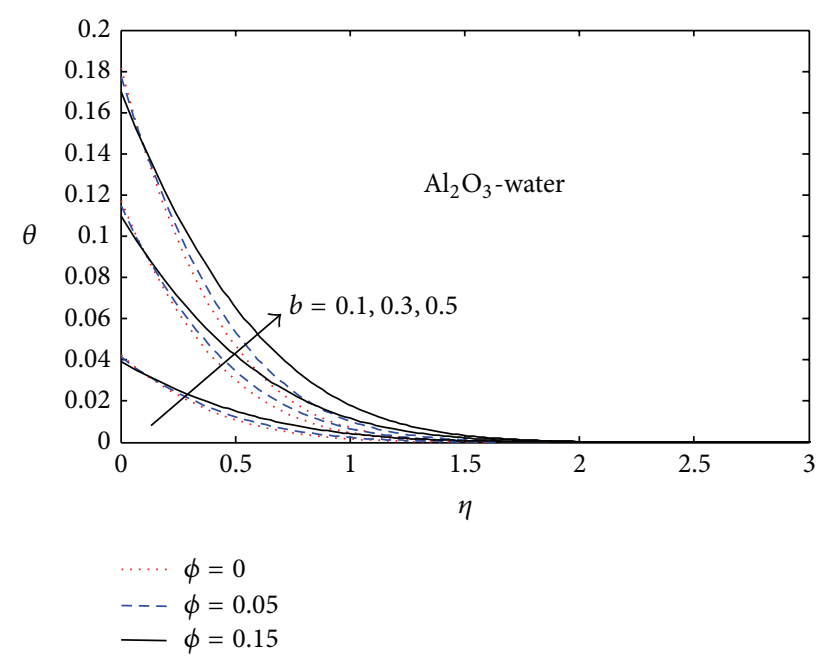

Figure $4: n=2, f_{w}=0.5, \operatorname{Pr}=6.2, M=0.5$, and $A=0.5$.

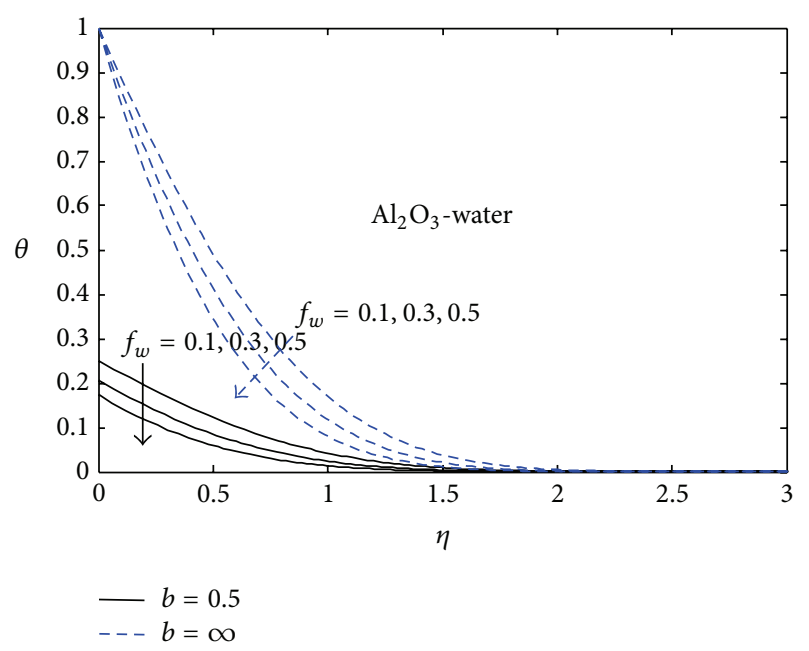

Figure $5: n=2, \phi=0.1, \operatorname{Pr}=6.2, M=0.5$, and $A=0.5$.

numerical computations, we plot the graphs given in Figures 2 to 10 , respectively.

To exhibit the effects of various values of the power law exponent $n$ on velocity $F^{\prime}(\eta)$ and temperature $\theta(\eta)$ for two values of the nanoparticles volume fraction $\phi$, which are $\phi=0$ (pure water) and $\phi \neq 0(=0.1)\left(\mathrm{Al}_{2} \mathrm{O}_{3}\right.$-water), keeping the other parameters fixed $(\operatorname{Pr}=6.2, M=0.5$, $b=0.5, f_{w}=0.5$, and $A=0.5$ ), we plot Figures 2 and 3 , respectively. It is clear from Figure 2 that the increased power law exponent increases the velocity. Also, it is clear that the presence of alumina nanoparticles reduces the momentum boundary layer thickness and increases the velocity. However, from Figure 3, it is seen that the increased power law exponent decreases the temperature and the existence of alumina nanoparticles increases the thermal boundary layer thickness. This is because the thermal conductivity of alumina is higher than that of water.

Figure 4 displays the influence of various values of the convective heat transfer parameter $b$ and the nanoparticles volume fractions $\phi$ on temperature distribution. We have

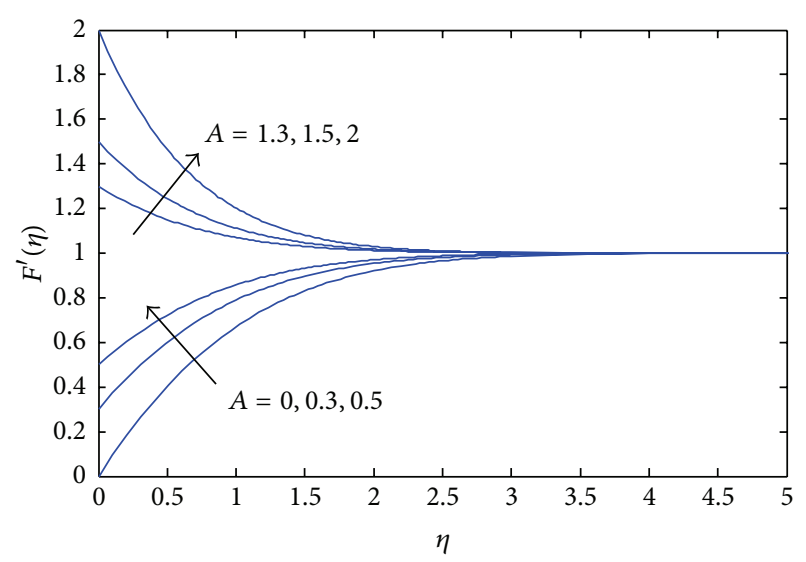

Figure $6: b=0.1, f_{w}=0.1, \operatorname{Pr}=6.2, M=0.5, n=2$, and $\phi=0.1$.

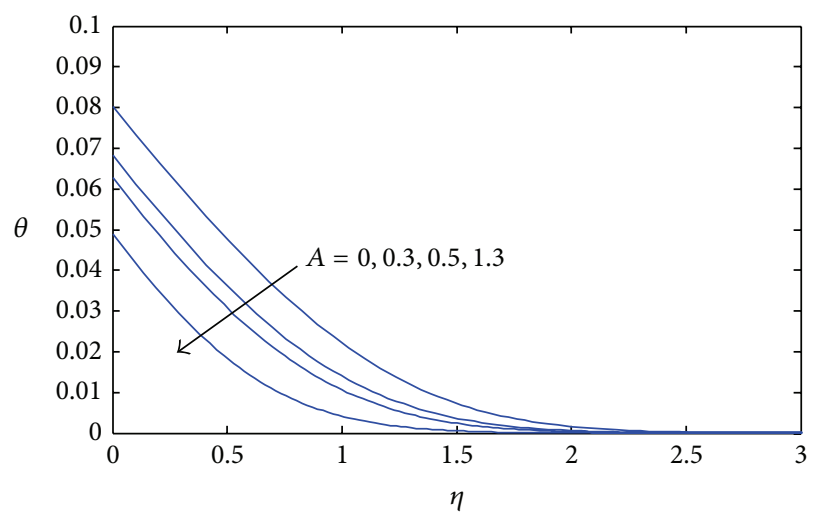

Figure $7: b=0.1, f_{w}=0.1, \operatorname{Pr}=6.2, M=0.5, n=2$, and $\phi=0.1$.

noted that the thermal boundary layer thickness increases when $n$ or $\phi$ increases. This agrees with the physical behavior that, when the convective heat transfer increases, the hot fluid side convection resistance decreases, and, consequently, the surface temperature increases (see Ishak [37]); because of this, the thermal boundary layer thickness increases, and because also alumina has high thermal conductivity, then the thickness increases more for alumina-water nanofluid.

Figure 5 shows the effect of the variation of suction parameter $f_{w}$ on temperature for two cases of convective heat transfer parameter $b$, first when $b=0.5$ (convective boundary condition) and second when $b=\infty$ (neglect convective condition, i.e., back to normal condition $\theta(0)=$ 1). All other parameters are nonzero. It can be seen that the thickness of the thermal boundary layer reduces with increasing values of $f_{w}$ for the two cases of $b$.

Effects of the velocity parameter $A$ on the velocity and temperature are shown in Figures 6 and 7, respectively. We have observed from Figure 6 that the velocity increases when $A$ increases. We have noticed also that for each $A<1\left(u_{0}<\right.$ $\left.U_{\infty}\right)$, the velocity increases till it becomes 1 and, for each $A>1\left(u_{0}>U_{\infty}\right)$ the velocity decreases till it becomes 1 while, from Figure 7 , it is noted that the temperature decreases when $A$ increases and the wall temperature also decreases when $A$ increases. 


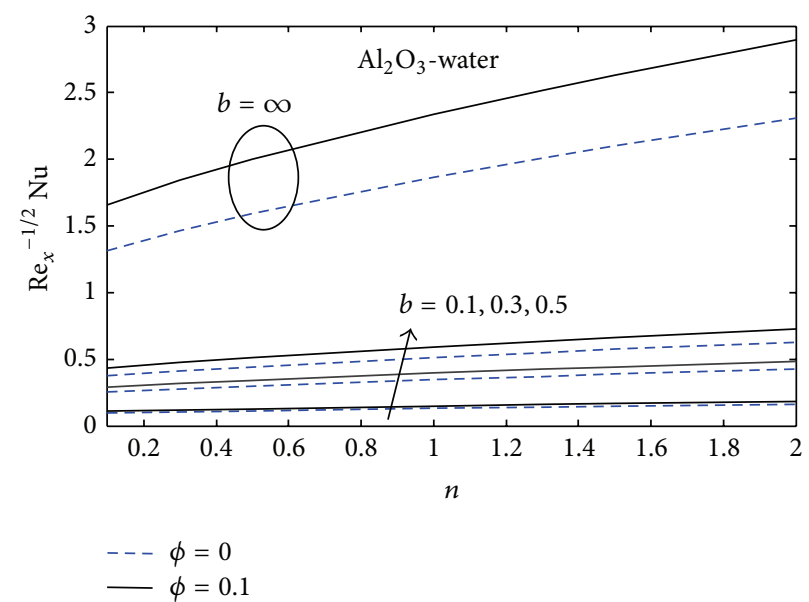

Figure 8: $\operatorname{Pr}=6.2, M=0.5, A=0.5$, and $f_{w}=0.1$.

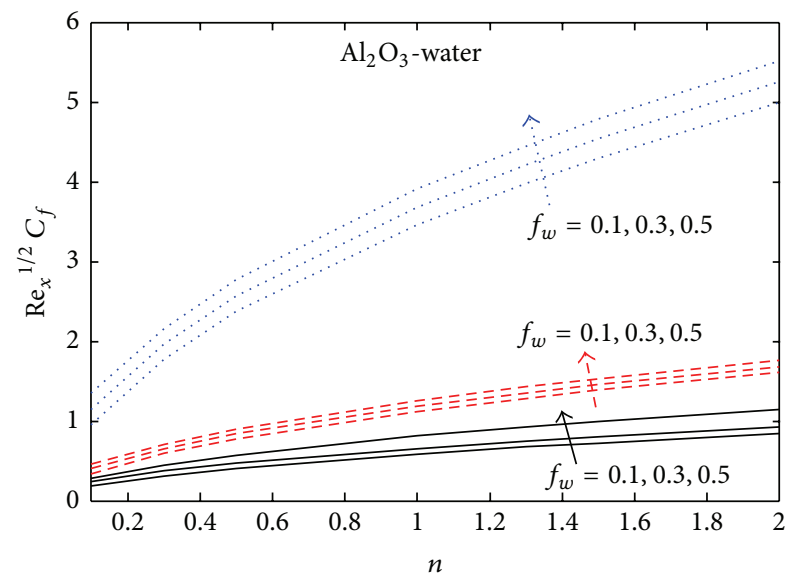

$$
\begin{aligned}
\text { … } A & =0.5 \\
--A & =0.7 \\
-A & =0.8
\end{aligned}
$$

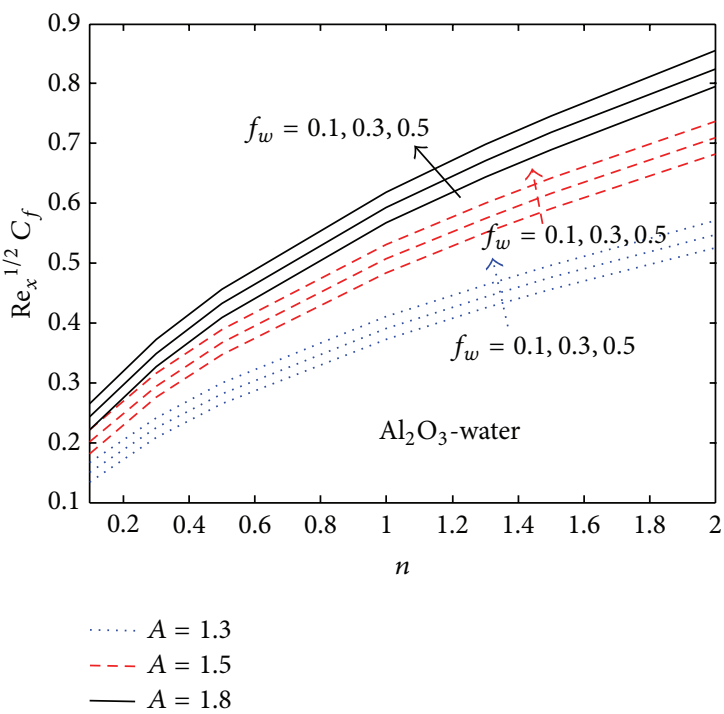

(b) $A>1$

(a) $A<1$

Figure 9: $\operatorname{Pr}=6.2, M=0.5, b=0.5$, and $\phi=0.1$.

Figure 8 shows the effects of $b$ on the shear stress versus the parameter $n$ for pure water and alumina-water nanofluid $(\phi \neq 0(=0.1))$. It is clear that the value of shear stress without considering the convictive boundary condition $(b=\infty)$ is greater than when considering the convective boundary condition for both $\phi=0$ and $\phi \neq 0$, also for the nanofluid the shear stress is greater more than for pure water. It is also noticed that increasing parameter $n$ leads to increasing the shear stress, as well as at each value of $n$, the shear stress be higher for higher value of $b$ both $\phi=0$ and $\phi \neq 0$.

Figures 9 and 10 show the effect of suction parameter $f_{w}$ and the velocity parameter $A$ on the shear stress and rate of heat transfer versus $n$, respectively. From Figure 9, we have seen that the shear stress increases when $f_{w}$ increases. For $A<1$ as in Figure 9(a), we have noted that the shear stress decreases when $A$ increases, but, for $A>1$ as in Figure 9(b), the shear stress increases with the increased $A$. However, from Figures 10(a) and 10(b), it is observed that the rate of heat transfer increases with the increasing value of suction parameter $f_{w}$ or the increasing value of the power law parameter $n$.

\section{Conclusion}

In this paper, we have studied the problem of heat transfer in MHD forced convection flow of alumina-water nanofluid over moving permeable vertical flat plate with convective surface boundary condition. New similarity transformations have been presented and solved numerically. It was found that alumina nanoparticles when suspended in a fluid are capable of increasing the heat transfer capability of the base fluid. The shear stress as well as heat transfer rate increases with nanoparticle volume fraction. Thermal boundary layer thickness as well as heat transfer rate increases when the convective heat transfer parameter $b$ increases. Thermal boundary layer thickness decreases while both shear stress 

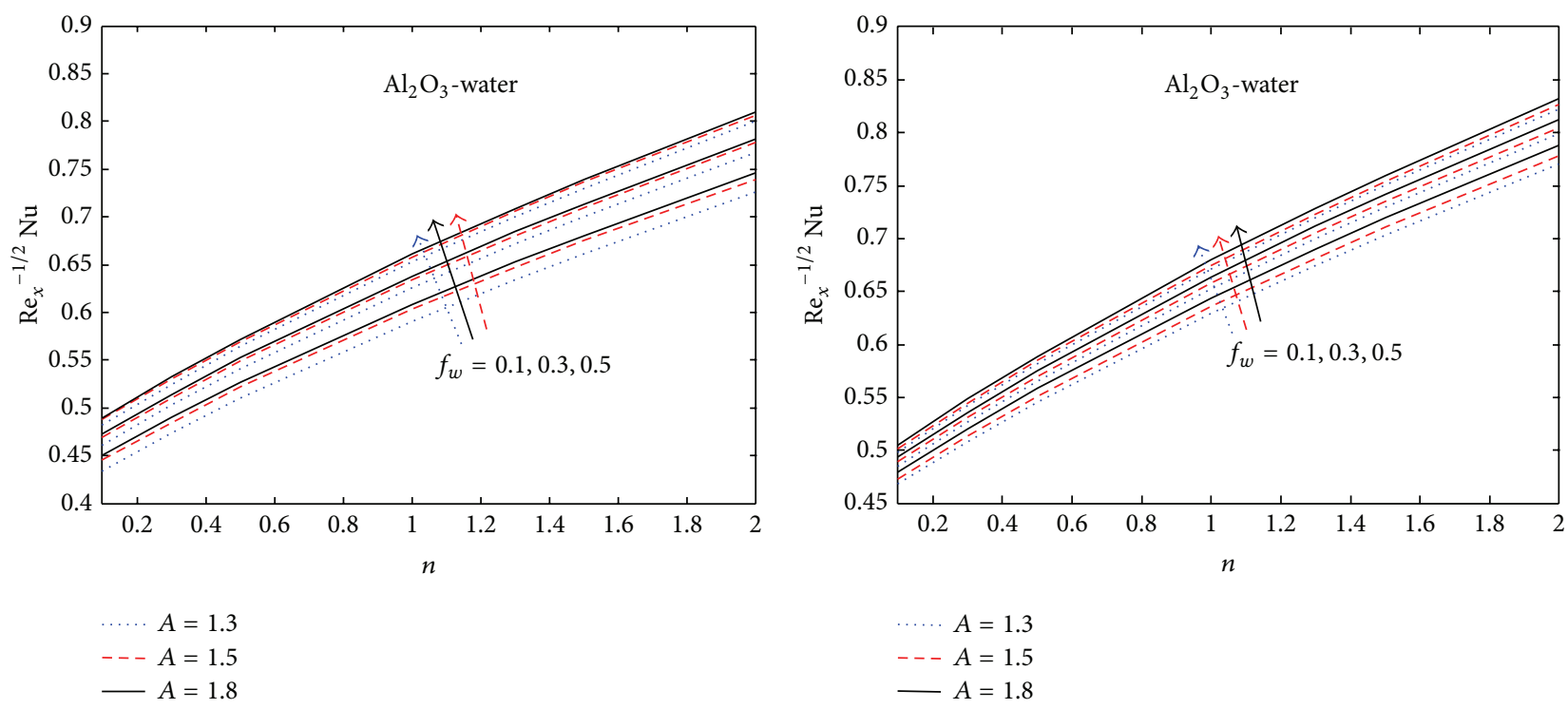

(a) $A<1$

(b) $A>1$

FIgURE 10: $\operatorname{Pr}=6.2, M=0.5, b=0.5$, and $\phi=0.1$.

and heat transfer rate increase with the increase of suction parameter. Temperature and wall temperature decrease while velocity and heat transfer rate increase when the velocity parameter $A$ increases.

\section{References}

[1] P. M. Congedo, S. Collura, and P. M. Congedo, "Modeling and analysis of natural convection heat transfer in nanofluids," in Proceedings of the ASME Summer Heat Transfer Conference (HT '09), vol. 3, pp. 569-579, August 2009.

[2] B. Ghasemi and S. M. Aminossadati, "Natural convection heat transfer in an inclined enclosure filled with a water-Cuo nanofluid," Numerical Heat Transfer A, vol. 55, no. 8, pp. 807823, 2009.

[3] C. J. Ho, M. W. Chen, and Z. W. Li, "Effect on natural convection heat transfer of nanofluid in an enclosure due to uncertainties of viscosity and thermal conductivity," in Proceedings of the ASME/JSME Thermal Engineering Summer Heat Transfer Conference (HT '07), vol. 1, pp. 833-841, July 2007.

[4] C. J. Ho, M. W. Chen, and Z. W. Li, "Numerical simulation of natural convection of nanofluid in a square enclosure: effects due to uncertainties of viscosity and thermal conductivity," International Journal of Heat and Mass Transfer, vol. 51, no. 1718, pp. 4506-4516, 2008.

[5] M. A. A. Hamad, I. Pop, and A. I. Md Ismail, "Magnetic field effects on free convection flow of a nanofluid past a vertical semi-infinite flat plate," Nonlinear Analysis: Real World Applications, vol. 12, no. 3, pp. 1338-1346, 2011.

[6] M. A. A. Hamad and I. Pop, "Scaling transformations for boundary layer stagnation-point flow towards a heated permeable stretching sheet in a porous medium saturated with a nanofluid and heat absorption/generation effects," Transport in Porous Media, vol. 87, no. 1, pp. 25-39, 2011.

[7] M. A. A. Hamad, "Analytical solution of natural convection flow of a nanofluid over a linearly stretching sheet in the presence of magnetic field," International Communications in Heat and Mass Transfer, vol. 38, no. 4, pp. 487-492, 2011.

[8] E. Abu-Nada and A. J. Chamkha, "Effect of nanofluid variable properties on natural convection in enclosures filled with a CuO-EG-Water nanofluid," International Journal of Thermal Sciences, vol. 49, no. 12, pp. 2339-2352, 2010.

[9] T. Teng, Y. Hung, T. Teng, H. Mo, and H. Hsu, "The effect of alumina/water nanofluid particle size on thermal conductivity," Applied Thermal Engineering, vol. 30, no. 14-15, pp. 2213-2218, 2010.

[10] C. J. Ho, W. K. Liu, Y. S. Chang, and C. C. Lin, "Natural convection heat transfer of alumina-water nanofluid in vertical square enclosures: an experimental study," International Journal of Thermal Sciences, vol. 49, no. 8, pp. 1345-1353, 2010.

[11] N. Bachok, A. Ishak, and I. Pop, "Boundary-layer flow of nanofluids over a moving surface in a flowing fluid," International Journal of Thermal Sciences, vol. 49, no. 9, pp. 1663-1668, 2010.

[12] F. M. Hady, F. S. Ibrahim, S. M. Abdel-Gaied, and M. R. Eid, "Effect of heat generation/absorption on natural convective boundary-layer flow from a vertical cone embedded in a porous medium filled with a non-Newtonian nanofluid," International Communications in Heat and Mass Transfer, vol. 38, no. 10, pp. 1414-1420, 2011.

[13] D. Lelea, "The performance evaluation of $\mathrm{Al}_{2} \mathrm{O}_{3}$ /water nanofluid flow and heat transfer in microchannel heat sink," International Journal of Heat and Mass Transfer, vol. 54, no. 1718, pp. 3891-3899, 2011.

[14] S. K. Nandy and T. R. Mahapatra, "Effects of slip and heat generation/absorption on MHD stagnation flow of nanofluid past a stretching/shrinking surface with convective boundary conditions," International Journal of Heat and Mass Transfer, vol. 64, pp. 1091-1100, 2013.

[15] D. Pal, G. Mandal, and K. Vajravelu, "MHD convectiondissipation heat transfer over a non-linear stretching and 
shrinking sheets in nanofluids with thermal radiation," International Journal of Heat and Mass Transfer, vol. 65, pp. 481-490, 2013.

[16] A. M. Rohni, S. Ahmad, and I. Pop, "Boundary layer flow over a moving surface in a nanofluid beneath a uniform free stream," International Journal of Numerical Methods for Heat and Fluid Flow, vol. 21, no. 7, pp. 828-846, 2011.

[17] S. K. Das, S. U. S. Choi, W. Yu, and T. Pradeep, Nanofluids: Science and Technology, Wiley, Hoboken, NJ, USA, 2007.

[18] X. Wang and A. S. Mujumdar, "Heat transfer characteristics of nanofluids: a review," International Journal of Thermal Sciences, vol. 46, no. 1, pp. 1-19, 2007.

[19] X. Wang and A. S. Mujumdar, "A review on nanofluids, part I: theoretical and numerical investigations," Brazilian Journal of Chemical Engineering, vol. 25, no. 4, pp. 613-630, 2008.

[20] X. Wang and A. S. Mujumdar, "A review on nanofluids, part II: experiments and applications," Brazilian Journal of Chemical Engineering, vol. 25, no. 4, pp. 631-648, 2008.

[21] S. Kakaç and A. Pramuanjaroenkij, "Review of convective heat transfer enhancement with nanofluids," International Journal of Heat and Mass Transfer, vol. 52, no. 13-14, pp. 3187-3196, 2009.

[22] M. E. Ali, "The effect of variable viscosity on mixed convection heat transfer along a vertical moving surface," International Journal of Thermal Sciences, vol. 45, no. 1, pp. 60-69, 2006.

[23] A. Ishak, R. Nazar, and I. Pop, "Boundary layer on a moving wall with suction and injection," Chinese Physics Letters, vol. 24, no. 8, pp. 2274-2276, 2007.

[24] E. Magyari, “The moving plate thermometer," International Journal of Thermal Sciences, vol. 47, no. 11, pp. 1436-1441, 2008.

[25] J. Hoernel, "On the similarity solutions for a steady MHD equation," Communications in Nonlinear Science and Numerical Simulation, vol. 13, no. 7, pp. 1353-1360, 2008.

[26] D. Pal and H. Mondal, "Influence of temperature-dependent viscosity and thermal radiation on MHD forced convection over a non-isothermal wedge," Applied Mathematics and Computation, vol. 212, no. 1, pp. 194-208, 2008.

[27] R. Kandasamy and I. Muhaimin, "Scaling transformation for the effect of temperature-dependent fluid viscosity with thermophoresis particle deposition on MHD-free convective heat and mass transfer over a porous stretching surface," Transport in Porous Media, vol. 84, no. 2, pp. 549-568, 2010.

[28] G. Herdricha, M. Auweter-Kurtza, M. Fertiga, A. Nawaza, and D. Petkowa, "MHD flow control for plasma technology applications," Vacuum, vol. 80, pp. 1167-1173, 2006.

[29] M. A. Seddeek, A. A. Afify, and M. A. Hanaya, "Similarity solutions for steady MHD Falkner-Skan flow and heat transfer over a wedge by considering the effect of variable viscosity and thermal conductivity," Applications and Applied Mathematics, vol. 4, pp. 301-313, 2009.

[30] M. S. Alam, M. M. Rahman, and M. A. Sattar, "Effects of variable suction and thermophoresis on steady MHD combined freeforced convective heat and mass transfer flow over a semiinfinite permeable inclined plate in the presence of thermal radiation," International Journal of Thermal Sciences, vol. 47, no. 6, pp. 758-765, 2008.

[31] O. Aydin and A. Kaya, "MHD mixed convection of a viscous dissipating fluid about a permeable vertical flat plate," Applied Mathematical Modelling, vol. 33, no. 11, pp. 4086-4096, 2009.

[32] M. M. Rahman and K. M. Salahuddin, "Study of hydromagnetic heat and mass transfer flow over an inclined heated surface with variable viscosity and electric conductivity," Communications in
Nonlinear Science and Numerical Simulation, vol. 15, no. 8, pp. 2073-2085, 2010.

[33] A. Aziz, "A similarity solution for laminar thermal boundary layer over a flat plate with a convective surface boundary condition," Communications in Nonlinear Science and Numerical Simulation, vol. 14, no. 4, pp. 1064-1068, 2009.

[34] H. F. Oztop and E. Abu-Nada, "Numerical study of natural convection in partially heated rectangular enclosures filled with nanofluids," International Journal of Heat and Fluid Flow, vol. 29, no. 5, pp. 1326-1336, 2008.

[35] H. Schlichting and K. Gersten, Boundary Layer Theory, McGraw-Hill, NewYork, NY, USA, 8th edition, 2000.

[36] W. M. Kays and M. E. Crawford, Convective Heat and Mass Transfer, McGraw Hill, New York, NY, USA, 4th edition, 2005.

[37] A. Ishak, "Similarity solutions for flow and heat transfer over a permeable surface with convective boundary condition," Applied Mathematics and Computation, vol. 217, no. 2, pp. 837842, 2010. 


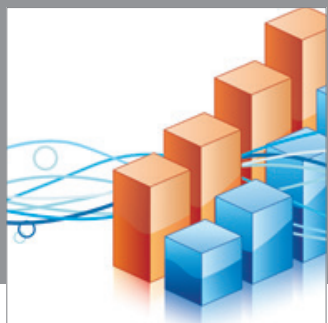

Advances in

Operations Research

mansans

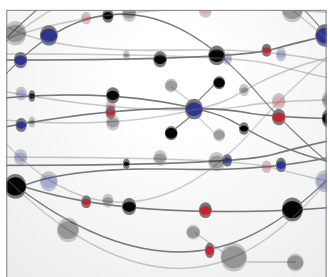

The Scientific World Journal
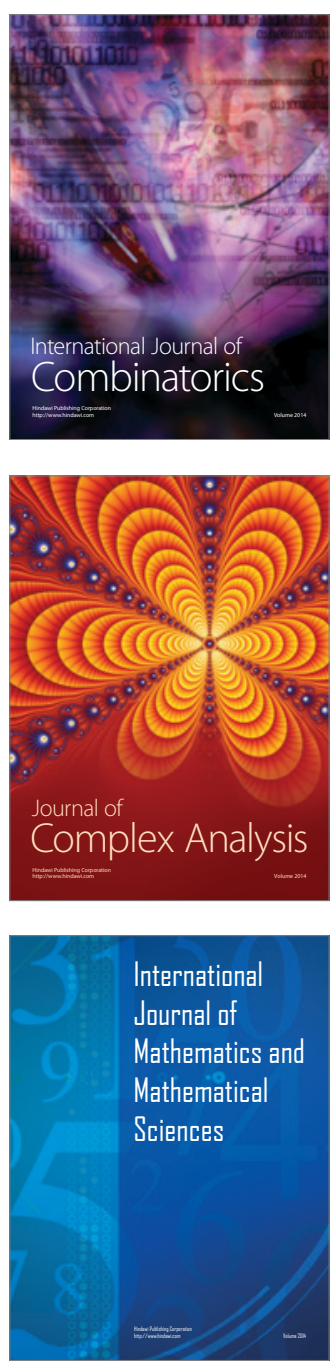
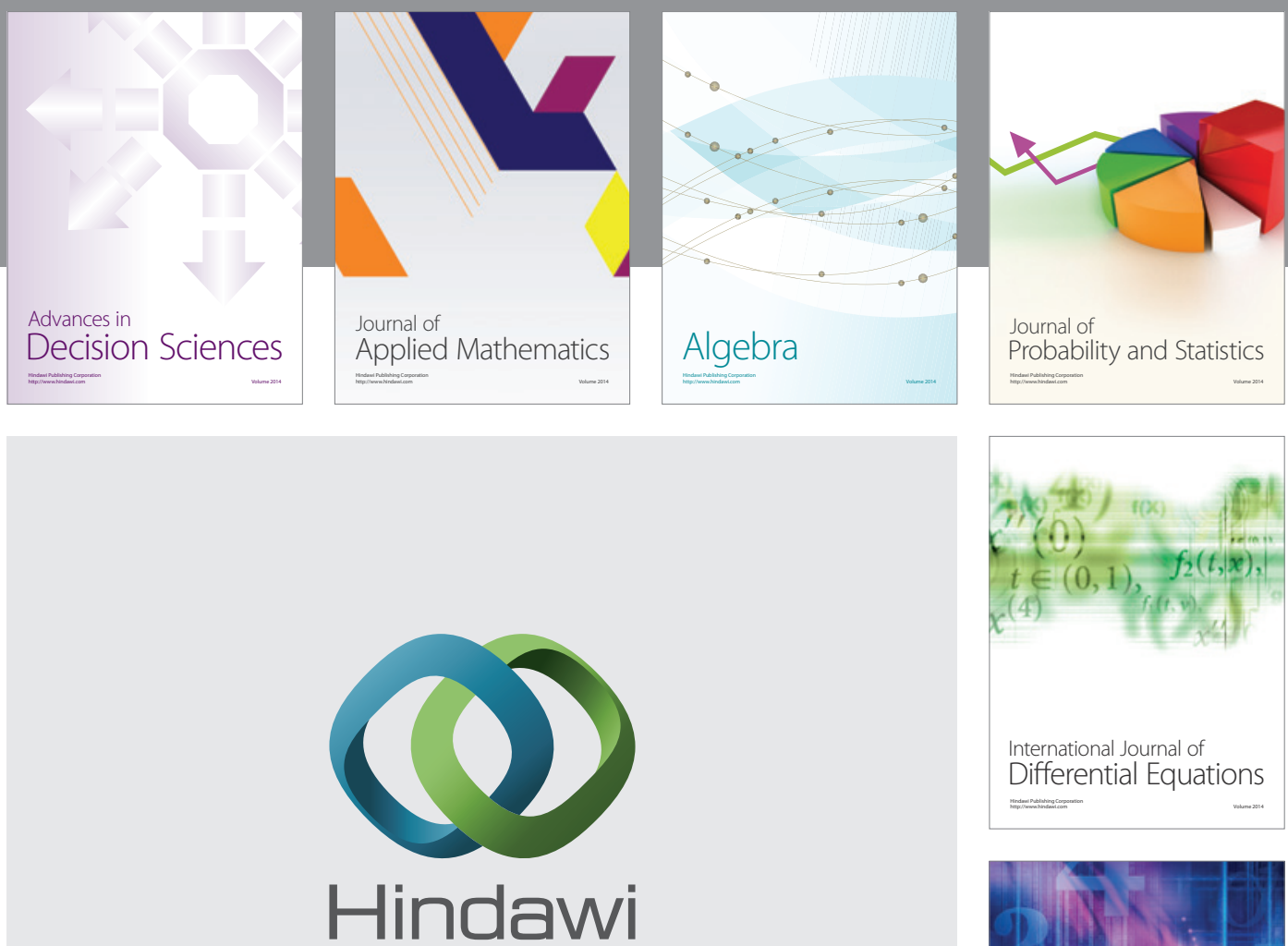

Submit your manuscripts at http://www.hindawi.com
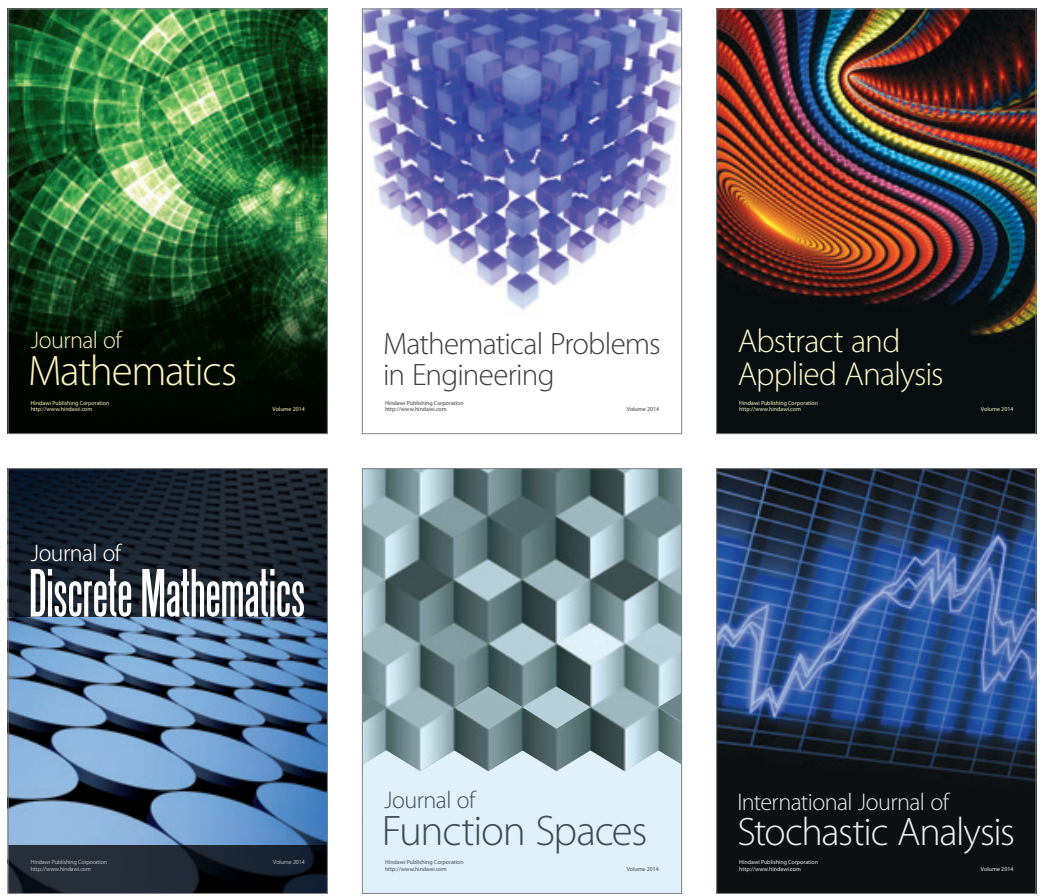

Journal of

Function Spaces

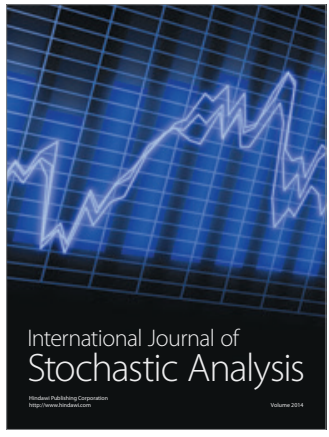

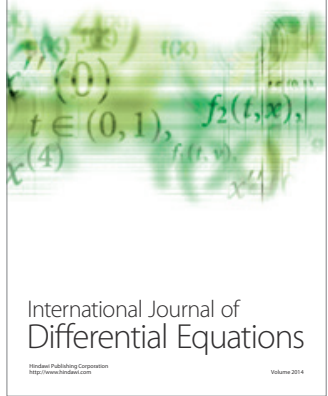
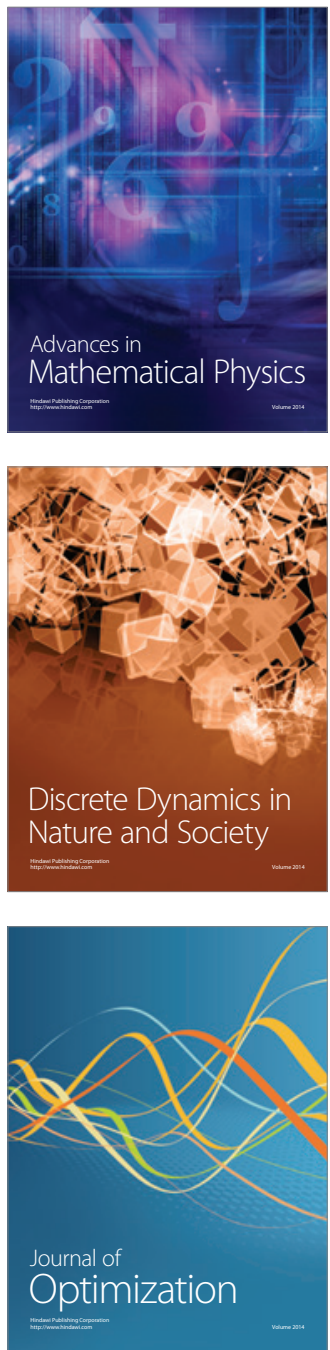